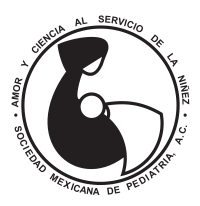

Vol. 87, No. 3 - Mayo-Junio 2020. pp 91-96 https://dx.doi.org/10.35366/94838

Revista Mexicana de

\title{
Factores de riesgo asociados a exanguinotransfusión por ictericia neonatal en un hospital universitario: estudio de casos y controles
}

\author{
Pablo Vásquez-Hoyos, ${ }^{\ddagger}, *$ Héctor Romero, ${ }^{\ddagger}$ Juan Pablo Álzate, ${ }^{\ddagger}$ Luis Hernando Riaño, ${ }^{\ddagger}$ \\ María Mercedes Góngora, ${ }^{\ddagger}$ Ricardo Andrés Roa ${ }^{\ddagger}$ \\ ‡Fundación Universitaria de Ciencias de la Salud. Hospital de San José de Bogotá, Colombia.
}

\begin{abstract}
RESUMEN
Introducción: La ictericia neonatal es una patología frecuente, la cual puede tener diferentes grados. En la actualidad, se conocen los factores asociados a hiperbilirrubinemia grave, pero no para la realización de exanguinotransfusión. Objetivo: Identificar factores asociados con la realización de exanguinotransfusión en recién nacidos $(\mathrm{RN})$ ictéricos, en una institución de Bogotá, Colombia. Material y métodos: Estudio de casos y controles, retrospectivo. Los casos fueron $\mathrm{RN}$ que requirieron exanguinotransfusión y los controles fueron RN ictéricos, pero sin este procedimiento. Los grupos fueron emparejados por sexo, edad gestacional, peso al nacimiento y tipo de incompatibilidad. Entre ambos grupos se compararon características demográficas y clínicas. Para la identificación de los factores de riesgo, se calcularon riesgos mediante un modelo de regresión logística. Resultados: Se incluyeron 50 casos y 198 controles. La tasa de exanguinotransfusión fue de 85 por 100,000 RN. Las variables asociadas a exanguinotransfusión fueron: alimentación suplementaria (OR 5.66 [IC 95\%; 1.25-25.67]), niveles menores de hematocrito (OR 0.88 [IC 95\%; 0.83-0.93]) y presencia de sepsis (OR 4.04 [IC 95\%; 1.21-13.5]). Conclusión: En esta población hubo alta frecuencia de exanguinotransfusiones. Los factores descritos pueden ayudar a identificar de manera oportuna $\mathrm{RN}$ con mayor riesgo.
\end{abstract}

Palabras clave: Ictericia neonatal, recién nacido, exanguinotransfusión de componentes sanguíneos.

\begin{abstract}
Introduction: Neonatal jaundice is a frequent pathology, which can have different degrees. Currently, the factors associated with severe hyperbilirubinemia are known, but not for the performance of exchange transfusion. Objective: To identify factors associated with exchange transfusion in jaundiced neonates, in Bogotá, Colombia. Material and methods: Retrospective case-control study. The cases were neonates who required exchange transfusion and the controls were icteric neonates without this procedure. Both groups were matched by sex, gestational age, birth weight, and type of blood incompatibility. Between both groups, demographic and clinical characteristics were compared. Risk factors were estimated using a logistic regression model. Results: Fifty cases and 198 controls were included. The exchange transfusion rate was 85 per 100,000 newborns. The variables associated to exchange transfusion were: supplementary feeding (OR 5.66 [95\% Cl; 1.25-25.67]), lower hematocrit levels (OR 0.88 [95\% Cl; 0.83-0.93]) and sepsis (OR 4.04 [IC $95 \% ; 1.21-13.5])$. Conclusion: In this population, there was a high frequency of exchange transfusions. Our findings can help to identify, in a timely manner, infants at higher risk.
\end{abstract}

Keywords: Neonatal jaundice, newborn, exchange whole blood transfusion.

\footnotetext{
* Correspondencia: PVH, pvasquez@fucsalud.edu.com

Conflicto de intereses: Los autores declaran que no tienen.

Citar como: Vásquez-Hoyos P, Romero H, Álzate JP, Riaño LH, Góngora MM, Roa RA. Factores de riesgo asociados a exanguinotransfusión por ictericia neonatal en un hospital universitario: estudio de casos y controles. Rev Mex Pediatr 2020; 87(3):91-96. doi: $10.35366 / 94838$

[Risk factors associated with exchange transfusion for neonatal jaundice in a university hospital: case-control study]
} 


\section{INTRODUCCIÓN}

La ictericia es producida por un incremento de los niveles de bilirrubinas en sangre, y constituye la patología más frecuente del periodo neonatal, ocurriendo en más de $60 \%$ de los recién nacidos $(\mathrm{RN}) .{ }^{1} \mathrm{Si}$ bien, existen múltiples etiologías que producen hiperbilirrubinemia, las cuales en su mayoría son fisiológicas, dada su alta prevalencia y su potencial para producir neurotoxicidad siempre debe tenerse en cuenta a fin de identificar neonatos en riesgo.

La bilirrubina no conjugada o indirecta tiene la propiedad de atravesar la barrera hematoencefálica y depositarse en tejido cerebral, generando manifestaciones neurológicas, denominadas BIND (disfunción neurológica inducida por bilirrubinas, por sus siglas en inglés) o secuelas neurológicas, en ocasiones fatales, conocidas como kernícterus. ${ }^{1-4}$

Para prevenir complicaciones relacionadas con la hiperbilirrubinemia, en especial en $\mathrm{RN}$ nacidos $>35$ semanas de edad gestacional, desde hace años se tienen disponibles protocolos de manejo. Así se define hiperbilirrubinemia grave, niveles séricos de bilirrubina total mayores al percentil 95, de acuerdo con las horas de vida, usando el nomograma de Bhutani. ${ }^{1}$

De acuerdo con la Academia Americana de Pediatría (AAP), el manejo inicial se basa en fototerapia y sólo se recomienda la exanguinotransfusión de forma inmediata en pacientes con síntomas neurológicos agudos, o bien, con hiperbilirrubinemia $>5 \mathrm{mg} / \mathrm{dL}$ ( 85 umol/L). ${ }^{2,3}$ Durante la exanguinotransfusión se busca reemplazar completamente la sangre del paciente mediante la extracción y administración de hemoderivados, con el fin de reducir agudamente el nivel de bilirrubinas. ${ }^{5,6}$

Aunque los factores de riesgo para hiperbilirrubinemia grave ya están descritos, ${ }^{7}$ los factores que permitan detectar pacientes que muy probablemente requerirán exanguinotransfusión no son claros. ${ }^{8}$ De esta forma, el objetivo de este trabajo fue identificar, en $\mathrm{RN}$ con hiperbilirrubinemia, los factores asociados a exanguinotransfusión, en una institución de Bogotá, Colombia.

\section{MATERIAL Y MÉTODOS}

Se realizó un estudio de casos y controles, con recolección de datos de manera retrospectiva en el Hospital de San José de Bogotá, Colombia, en el periodo comprendido entre enero de 2013 a julio de 2018. Se seleccionaron pacientes $>35$ semanas de gestación, con diagnóstico de ictericia neonatal. El grupo de casos fueron los pacientes en quienes se realizó exanguinotransfusión, tomando los criterios de la AAP; mientras que el grupo control se constituyó con quienes no se les realizó este procedimiento. Los casos y controles fueron pareados por sexo, edad gestacional, tipo de incompatibilidad (Rh, ABO, doble y sin incompatibilidad) y peso al nacer $(<2,500 \mathrm{~g}, 2,500-3,000 \mathrm{~g}, 3,000-3,500 \mathrm{~g}$ y $>3,500 \mathrm{~g})$, en una razón de cuatro controles por caso. Se excluyeron pacientes con hiperbilirrubinemia conjugada o trastornos metabólicos por déficit enzimáticos.

La selección de los controles fue mediante un muestreo no aleatorio, consecutivo. Se revisaron los expedientes clínicos para identificar los factores de riesgo asociados a la exanguinotransfusión, como características de los padres (edad, nivel educativo), lactancia materna exclusiva, sepsis, deshidratación hipernatrémica, y trauma en periparto. También se buscaron factores asociados a la atención como tipo de aseguramiento (contribuyente o subsidiado), acudir a consulta al egreso del hospital, la toma de bilirrubinas en las primeras 12 horas, tratamiento de la ictericia con fototerapia, así como variables relacionadas a hemolisis (Coombs, hemoglobina, niveles de reticulocitos y esquistocitos).

Análisis estadístico. Las variables cuantitativas se presentan como promedio \pm desviación típica. Las cualitativas con números absolutos y porcentajes. Para la comparación de las variables cuantitativas entre los grupos se utilizó prueba t-Student y para las variables cualitativas la prueba de $\chi^{2}$. Las variables que fueron estadísticamente significativas, se integraron en un modelo de regresión logística, realizando análisis de pruebas de bondad de ajuste con la prueba de Hosmer-Lemeshow. Valores de $p<$ 0.05 se consideraron estadísticamente significativos. Los análisis de datos fueron realizados con el paquete estadístico STATA 13.

Aspectos éticos. Esta investigación se clasificó como investigación sin riesgo, y fue evaluada y aprobada por el Comité de Ética de Investigación de la institución.

\section{RESULTADOS}

Durante el periodo de estudio se detectaron 50 pacientes que se realizaron exanguinotransfusión, a partir de un total de 23,425 RN > 35 semanas de gestación. Así la incidencia fue de 85 casos por $100,000 \mathrm{RN}$ vivos.

Cabe señalar que en 11 casos $(22 \%)$ no se contaba con la determinación de bilirrubinas a las 12 horas de vida. En nueve (81\%) porque no hubo incompatibilidad 


\begin{tabular}{|c|c|c|}
\hline Variable & $\begin{array}{c}\text { Casos }(n=50) \\
n(\%)\end{array}$ & $\begin{array}{c}\text { Controles }(n=198) \\
n(\%)\end{array}$ \\
\hline EG (semanas)* & $37.9 \pm 1.3$ & $38.0 \pm 1.2$ \\
\hline Peso al nacer (gramos)* & $3,013 \pm 441$ & $2,938 \pm 442$ \\
\hline \multicolumn{3}{|l|}{ Sexo } \\
\hline Masculino & $20(40.0)$ & $84(42.4)$ \\
\hline Femenino & $30(60.0)$ & $114(57.6)$ \\
\hline \multicolumn{3}{|l|}{ Tipo de incompatibilidad } \\
\hline Doble incompatibilidad & $2(4.0)$ & $7(3.5)$ \\
\hline Incompatibilidad de grupo únicamente & $23(46.0)$ & $111(56.1)$ \\
\hline Incompatibilidad de Rh únicamente & $13(26.0)$ & $32(16.2)$ \\
\hline Ninguna incompatibilidad & $12(24.0)$ & $48(24.2)$ \\
\hline
\end{tabular}

y en dos pacientes, la medición se omitió por el médico tratante. Estos 11 pacientes fueron detectados posterior al egreso; tres regresaron porque los padres identificaron la ictericia, el resto fueron enviados después de una valoración médica, durante el control postnatal programado.

En la Tabla 1 se compararon los 50 casos, con el grupo de 198 controles; como se observa, la distribución de las variables que se utilizaron para emparejar los grupos fue apropiada.

En la Tabla 2 se muestra el análisis univariado de los factores estudiados asociados a exanguinotransfusión. Se observa que pertenecer al régimen contributivo, recibir lactancia materna exclusiva y la frecuencia de consultas fue mayor en el grupo control.

En cuanto al nivel de bilirrubinas, fue estadísticamente mayor en los casos $(13.87 \pm 5.19 \mathrm{mg} / \mathrm{dL}$ vs $8.18 \pm 3.18 \mathrm{mg} / \mathrm{dL} ; \mathrm{p}<0.0001)$, lo mismo ocurrió con los signos de hemólisis, tanto por la intensidad en el Coombs $(p=0.008)$, como por el valor promedio de reticulocitos $(11.1 \pm 8.3 \%$ vs $7.3 \pm 3.6 \%$; < 0.001$)$ y el menor nivel de hemoglobina $(15.1 \pm 3.5 \mathrm{mg} / \mathrm{dl} \mathrm{vs}$ $17.9 \pm 2.4 \mathrm{mg} / \mathrm{dl}, \mathrm{p}<0.001)$. El otro factor asociado con exanguinotransfusión fue la presencia de sepsis (18 vs $5.5 \% ; p=0.004)$

Como también se muestra en la Tabla 2, no hubo diferencias para deshidratación hipernatrémica, presencia de cefalohematomas o trauma periparto.

Al realizar el análisis de regresión logística (Tabla 3) se evidenció que la necesidad de alimentación suplementaria en las primeras horas de vida (OR 5.66; IC 95\% [1.25 a 25.67]), sepsis (OR 4.04; IC 95\% [1.21 a 13.50]) y menores niveles de hematocrito (OR 0.88; IC 95\% [0.83-0.93]) fueron factores independientes asociados a exanguinotransfusión.

\section{DISCUSIÓN}

Un primer hallazgo de este estudio fue que parece haber una alta incidencia de procedimientos de exanguinotransfusión. En comparación al reporte de Bhutani en 2016 quien, al analizar aproximadamente 90\% de los centros hospitalarios de California, calculó la tasa de incidencia en 3.4 procedimientos por $100,000 \mathrm{RN}>35$ semanas, ${ }^{5}$ lo cual es muy inferior a lo observado por nosotros. Sin embargo, nuestro hospital atiende pacientes de alta complejidad y el otro reporte corresponde a una incidencia poblacional. En contraste, en un hospital en la India en el 2015, se reporta una incidencia de 120 casos por $100,000 \mathrm{RN}^{7}$ Siendo este valor más cercano al de 85 por 100,000 de nuestro hospital, pero es posible que sea mayor porque incluyeron $\mathrm{RN}<35$ semanas.

En diferentes estudios se han identificado los factores de riesgo para hiperbilirrubinemia grave, como incompatibilidad aloinmune o hemolítica, deficiencia de glucosa 6 fosfato deshidrogenasa, historia de hermanos con ictericia neonatal, cefalohematoma y lactancia materna exclusiva. ${ }^{5,7}$ Sin embargo, sobre factores específicos relacionados con exanguinotransfusión sólo existen estudios descriptivos, en los cuales se ha identificado principalmente a la prematuridad y la incompatibilidad ABO. ${ }^{7-12}$ Estos dos factores no 
fueron analizados en este estudio, ya que se usaron como parear los dos grupos estudiados.

Maisels y Kring en 1998 describen que, de un total de 29,974 RN, reingresaron a un hospital 247 (0.8\%). Reportan que cursar con ictericia en las primeras horas de nacimiento, la menor edad gestacional y la lactancia materna se asociaron a ingresar por hiper- bilirrubinemia. ${ }^{11}$ En este estudio también comprobamos el posible efecto de la lactancia, pero creemos esto es más bien un reflejo de la pobre ingesta de los pacientes.

Por otro lado, la sepsis representa $12 \%$ de los casos de hiperbilirrubinemia grave en la literatura y está descrito como la causa más común para la exanguino-

Tabla 2: Análisis univariado de los factores asociados a exanguinoneotransfusión.

\begin{tabular}{|c|c|c|c|}
\hline & $\begin{array}{c}\text { Casos }(n=50) \\
n(\%)\end{array}$ & $\begin{array}{c}\text { Controles }(n=198) \\
n(\%)\end{array}$ & $\mathrm{p}^{*}$ \\
\hline Edad materna (años) ${ }^{\star *}$ & $29.2 \pm 6.64$ & $28.18 \pm 6.42$ & 0.318 \\
\hline Régimen contributivo & $43(86.0)$ & $193(97.5)$ & 0.003 \\
\hline \multicolumn{4}{|l|}{$\begin{array}{l}\text { Nivel educativo de la madre } \\
(\mathrm{NCa}=47 / \mathrm{NC}=193)\end{array}$} \\
\hline Técnico/profesional & $15(32.0)$ & $74(38.0)$ & \\
\hline Bachiller & $23(48.9)$ & $92(47.7)$ & 0.576 \\
\hline Sin estudios completos & $9(19.9)$ & $27(14.0)$ & \\
\hline \multicolumn{4}{|l|}{$\begin{array}{l}\text { Nivel educativo del padre } \\
(\mathrm{NCa}=47 / \mathrm{NC}=193)\end{array}$} \\
\hline Técnico/profesional & $13(27.7)$ & $48(24.9)$ & \\
\hline Bachiller & $21(44.7)$ & $107(55.4)$ & 0.356 \\
\hline Sin estudios completos & $13(27.7)$ & $38(19.7)$ & \\
\hline Lactancia materna exclusiva & $43(86.0)$ & $192(97.0)$ & 0.002 \\
\hline Acudir a consulta antes de hospitalización & $3(6.0)$ & $41(20.7)$ & 0.013 \\
\hline \multicolumn{4}{|l|}{ Bilirrubinas a las 12 horas de vida } \\
\hline Se tomaron & $39(78.0)$ & $173(87.4)$ & 0.093 \\
\hline Niveles (mg/dL) medio** & $13.87 \pm 5.19$ & $8.18 \pm 3.18$ & $<0.001$ \\
\hline \multicolumn{4}{|l|}{ Indicación inicial } \\
\hline Exanguinotransfusión inmediata & $20(40.0)$ & $0(0.0)$ & \\
\hline Fototerapia intensiva & $24(80.0)$ & $44(22.2)$ & $<0.001$ \\
\hline Fototerapia simple & $3(10.0)$ & $98(49.5)$ & \\
\hline Egreso & $3(10.0)$ & $56(28.3)$ & \\
\hline \multicolumn{4}{|l|}{ Coombs positivo ( $\mathrm{NCa}=30 / \mathrm{NCo}=82$ ) } \\
\hline+ & $4(13.3)$ & $23(28.0)$ & \\
\hline++ & $9(30.0)$ & $34(41.3)$ & 0.008 \\
\hline+++ & $11(36.7)$ & $23(28.0)$ & \\
\hline++++ & $6(20.0)$ & $2(2.4)$ & \\
\hline Reticulocitos $(\mathrm{NCa}=46 / \mathrm{NCo}=165)^{* *}$ & $11.1 \pm 8.3$ & $7.3 \pm 3.6$ & $<0.001$ \\
\hline Esquistocitos (NCa=45/NCo=165) & $14(31.1)$ & $41(24.8)$ & 0.397 \\
\hline Nivel de hemoglobina** & $15.1 \pm 3.5$ & $17.9 \pm 2.4$ & $<0.001$ \\
\hline Nivel de hematocrito** & $45.5 \pm 10.1$ & $54.9 \pm 7.5$ & $<0.001$ \\
\hline Sepsis & $9(18.0)$ & $11(5.5)$ & 0.004 \\
\hline Deshidratación hipernatrémica & $3(6.0)$ & $4(2.0)$ & 0.586 \\
\hline Cefalohematoma/trauma periparto & $0(0.0)$ & $5(2.5)$ & 0.148 \\
\hline
\end{tabular}




\begin{tabular}{lllc} 
& OR & IC 95\% & P \\
\hline Alimentación suplementaria & 5.66 & $1.25-25.67$ & 0.02 \\
Menores niveles de hematocrito & 0.88 & $0.83-0.93$ & 0.02 \\
Sepsis & 4.04 & $1.21-13.50$ & 0.09 \\
Régimen contributivo & 0.27 & $0.06-1.24$ & 0.20 \\
Acudir a consulta antes hospitalización & 0.42 & $0.11-1.61$ & 0.67 \\
Coombs negativo & (Ref) & 0.57 \\
$\quad+$ & 0.75 & $0.19-2.88$ & 0.55 \\
$\quad++$ & 0.74 & $0.26-2.11$ & 0.11 \\
$\quad+++$ & 1.36 & $0.48-3.82$ & $0.67-47.89$ \\
\hline OR = razón de momios; IC 95\% = intervalo de confianza del $95 \%$. & 5.68 & &
\end{tabular}

transfusión, ${ }^{12,13}$ datos que coinciden con los hallazgos del presente estudio.

La identificación del sistema de seguridad social contributivo (personas que aportan económicamente al sistema de salud) como un factor protector. Es posible que un régimen diferente podría llevar a demorar la atención del RN; sin embargo, esta diferencia no se logró confirmar en el análisis multivariado, seguramente por el bajo número de sujetos pertenecientes a otros regímenes.

Es llamativo el elevado número de casos que presentan algún tipo de incompatibilidad, lo que sugiere que esta población se beneficiaría de estrategias de tamizaje universal no invasivo como parte del protocolo institucional, previo al egreso del neonato. Esta estrategia ha mostrado ser efectivo para la identificación de pacientes con mayor riesgo de hiperbilirrubinemia. ${ }^{14-17}$

Los resultados de este estudio tienen que contextualizarse con respecto a sus fortalezas y limitaciones. La principal fortaleza de este estudio es que incluyeron un gran número de casos de pacientes que requirieron exanguinotransfusión; mientras que las limitaciones incluyen la recolección retrospectiva basada en registros clínicos, que genera un sesgo de información, ya que algunos datos pudieron ser omitidos o no realizados. También existen sesgos en la selección de los controles (dado que no fue aleatoria) y de los casos, ya que al verificar los criterios de exanguinotransfusión, muchos no cumplieron los criterios sugeridos por la AAP. Finalmente, es posible que la validez externa se vea comprometida dado que este es un hospital de referencia.

\section{AGRADECIMIENTOS}

Expresamos nuestro agradecimiento al Servicio de Pediatría del Hospital de San José y a la División de Investigaciones de la Fundación Universitaria de Ciencias de la Salud (FUCS).

\section{REFERENCIAS}

1. Maisels MJ. Physiologic and pathologic jaundice: the conundrum of defining normal bilirubin levels in the newborn. Pediatrics. 2006; 118(2): 805-807.

2. Johnson LH, Bhutani VK, Brown AK. System-based approach to management of neonatal jaundice and prevention of kernicterus. J Pediatr. 2002; 140(4): 396-403.

3. Newman TB, Liljestrand P, Escobar GJ. Infants with bilirubin levels of $30 \mathrm{mg} / \mathrm{dL}$ or more in a large managed care organization. Pediatrics. 2003; 111(6 Pt 1): 1303-1311.

4. Newman TB, Liljestrand P, Escobar GJ. Combining clinical risk factors with serum bilirubin levels to predict hyperbilirubinemia in newborns. Arch Pediatr Adolesc Med. 2005; 159(2): 113-119.

5. Bhutani VK, Meng NF, Knauer Y, Danielsen BH, Wong RJ, Stevenson DK et al. Extreme hyperbilirubinemia and rescue exchange transfusion in California from 2007 to 2012. J Perinatol. 2016; 36(10): 853-857. doi: 10.1038/jp.2016.106.

6. Flaherman VJ, Kuzniewicz MW, Escobar GJ, Newman TB. Total serum bilirubin exceeding exchange transfusion thresholds in the setting of universal screening. J Pediatr. 2012; 160(5): 796-800.e1.

7. Bujandric N, Grujic J. Exchange transfusion for severe neonatal hyperbilirubinemia: 17 years' experience from Vojvodina, Serbia. Indian J Hematol Blood Transfus. 2015; 32(2): 208-214. doi: 10.1007/s12288-015-0534-1.

8. Bhutani VK, Johnson L, Sivieri EM. Predictive ability of a predischarge hour-specific serum bilirubin for subsequent significant hyperbilirubinemia in healthy term and near-term newborns. Pediatrics. 1999; 103(1): 6-14.

9. Ballot DE, Rugamba G. Exchange transfusion for neonatal hyperbilirubinemia in Johannesburg, South Africa, from 
2006 to 2011. Int Sch Res Notices. 2016; 1-5. doi: 10.1155/2016/1268149.

10. Davutoğlu M, Garipardiç $M$, Güler E, Karabiber H, Erhan D. The etiology of severe neonatal hyperbilirubinemia and complications of exchange transfusion. Turk J Pediatr. 2010; 52(2): 163-166.

11. Maisels MJ, Kring E. Length of stay, jaundice, and hospital readmission. Pediatrics. 1998; 101(6): 995-998.

12. AmericanAcademy of Pediatrics Subcommittee on Hyperbilirubinemia. Management of hyperbilirubinemia in the newborn infant 35 or more weeks of gestation. Pediatrics. 2004; 114(1): 297-316.

13. Gamaleldin R, Iskander I, Seoud I, Aboraya H, Aravkin A, Sampson $P D$ et al. Risk factors for neurotoxicity in newborns with severe neonatal hyperbilirubinemia. Pediatrics. 2011; 128(4): e925-e931. doi: 10.1542/peds.2011-0206.
14. Eggert LD, Wiedmeier SE, Wilson J, Christensen RD. The effect of instituting a prehospital-discharge newborn bilirubin screening program in an 18-hospital health system. Pediatrics. 2006; 117(5): e855-e862.

15. Kuzniewicz MW, Escobar GJ, Newman TB. Impact of universal bilirubin screening on severe hyperbilirubinemia and phototherapy use. Pediatrics. 2009; 124(4): 1031-1039.

16. Jonguitud-Aguilar J, Noyola-Salazar CA, De Jesús-Raya E, Montes-Acuña OJ. Detección de ictericia neonatal durante la visita para tamiz metabólico neonatal. Rev Mex Pediatr. 2018; 85: 212-215.

17. Mah MP, Clark SL, Akhigbe E, Englebright J, Frye DK, Meyers JA et al. Reduction of severe hyperbilirubinemia after institution of predischarge bilirubin screening. Pediatrics. 2010; 125(5): e1143-e1148. 